\title{
MULTIPLE COLONIC AND GASTRIC METASTASIS OF ENDOMETRIAL ADENOCARCINOMA - CASE REPORT
}

\author{
C. Molnar ${ }^{1}$, Ecaterina Daniela Dobru ${ }^{2}$, C. Copotoiu ${ }^{1}$, C. Silaghi ${ }^{1}$, V.I Neagoe ${ }^{1}$, \\ Doina Milutin $^{3}$, A. Panţîru ${ }^{1}$, C.V. Molnar ${ }^{4}$ \\ 1) Surgical Clinic 1, Emergency Clinical County Hospital Târgu Mureş, Romania \\ 2) Gastroenterology Clinic 1, Emergency Clinical County Hospital Târgu Mureş, Romania \\ 3) Pathology Department, Emergency Clinical County Hospital Târgu Mureş, Romania \\ 4) Obstetrics-Gynecology Clinic 1, Emergency Clinical County Hospital Târgu Mureş, Romania
}

\begin{abstract}
MULTIPLE COLONIC AND GASTRIC METASTASIS OF ENDOMETRIAL ADENOCARCINOMA - CASE REPORT (Abstract): INTRODUCTION: In the gynecological malignancies endometrial adenocarcinoma is the second most common, giving early hematogenous and lymphatic metastasis to the lungs, brain and bones. CASE REPORT: We present the case of a 71 year old patient who underwent surgery two years ago for endometrial adenocarcinoma and followed postoperatively multimodal oncological treatment. The patient was admitted in January 2013 in the First Surgery Clinic of the Emergency Clinical County Hospital Târgu Mureş, Romania, for chronic anemia, consumptive disease signs and subocclusive syndrome. The preoperative exams (upper and lower gastrointestinal endoscopy, thoracoabdominal computed tomography and ultrasound) found a tumor invading right, transverse and sigmoid colon as well as gastric antrum and spleen. A subtotal colectomy with ileo-rectal anastomosis was then performed associated with distal gastrectomy and splenectomy. The histopathological exams revealed gastric and colonic metastasis from the endometrial serous cancer operated two years ago. The postoperative evolution was uneventful. CONCLUSIONS: Endometrial cancer can metastasize to the colon and stomach even without the presence of endometriosis. Multivisceral resections and preoperative exams of the digestive tract are justified in terms of subjective complaints of an occlusive simptomatology.
\end{abstract}

KEY WORDS: ENDOMETRIAL ADENOCARCINOMA; COLONIC METASTASIS; GASTRIC METASTASIS; SUBTOTAL COLECTOMY; MULTIVISCERAL RESECTIONS

SHORT TITLE: Colonic and gastric metastasis from endometrial adenocarcinoma

HOW TO CITE: Molnar C, Dobru ED, Copotoiu C, Silaghi C, Neagoe VI, Milutin D, Panţîru A, Molnar CV. Multiple colonic and gastric metastases of endometrial adenocarcinoma - case report. Jurnalul de chirurgie (Iaşi). 2013; 9(4): 339343. DOI: 10.7438/1584-9341-9-4-6.

\section{INTRODUCTION}

Among gynecologic malignancies, endometrial adenocarcinoma is the second most common, with early lymphatic and hematogenous metastasis [1,2]. Hematogenous dissemination is frequently noted in lungs, brain and bones [3]. Metastasis of endometrial adenocarcinoma in the colon are cited in the literature, but in most cases are associated with endometriosis [4,5]. Isolated splenic metastasis are reported in literature [6]. Our observation shows the possibility, exceptionally rare, of multiple metastasis of endometrial adenocarcinoma to the colon (ascending, transverse and descending colon) and to the stomach (gastric antrum) invading the lower pole of the spleen.

\section{CASE REPORT}

We present the case of a 71 year old woman who underwent in 2011 a radical 
lymphadeno-hystero-colpectomy (Wertheim operation) for endometrial serous adenocarcinoma noted as stage IIIb $\left(\mathrm{pT}_{3 \mathrm{~b}} \mathrm{~N}_{1} \mathrm{M}_{0}\right)$ according to the 2009 FIGO (International Federation of Gynecology and Obstetrics) staging [7]. The patient received postoperatively radio- and chemotherapy. The initially favorable evolution (no signs of local recurrence and distant determinations) was complicated two years postoperatively with impaired general condition, subocclusive syndrome (intermittent abdominal pain, constipation alternating with diarrhea), weight loss (6 $\mathrm{kg}$ in 3-4 months) and chronic anemia.

The reevaluation in the oncology service revealed at the abdominal ultrasound, multiple tumors situated on the right flank and left upper quadrant of the abdomen, adjacent to the intestinal loops and multiple lymph nodes up to $14 \mathrm{~mm}$ in diameter in the proximity of the superior mesenteric artery. The tumor specific antigens also markedly increased: CA-125: 40.6 u/mL, CA-19.9: 83.2 UI/mL, CEA: 12 $\mathrm{ng} / \mathrm{mL}$.

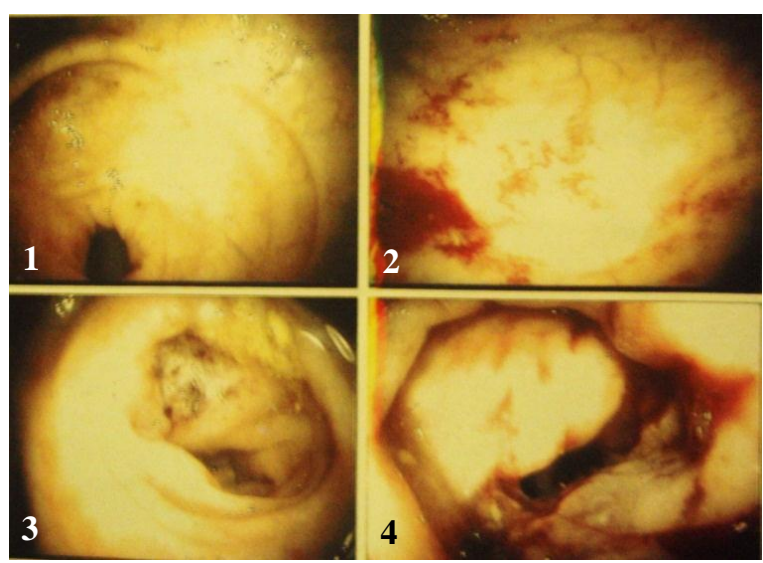

Fig. 1 Endoscopic findings: 1) ileocecal valve, 2) transverse colon, 3) right colon with ulcerated tumor, 4) sigmoid colon with ulcerated tumor

The lower gastrointestinal endoscopy revealed in the ascending colon the presence of an infiltrative hemi circumferential tumor with friable mucosa and bleeding on touch, which can't be exceeded by the endoscope (Fig. 1). A deep ulcer of $1.5 \mathrm{~cm}$ in diameter is discovered in the loop of the sigmoid colon, covered with fibrin as well as rectal angiectasia probably related to radiation proctitis.

The histopathological findings of the biopsies have revealed the diagnosis of serous adenocarcinoma metastasis, with a microscopic appearance being similar to the endometrial adenocarcinoma for which the patient underwent surgery in the past.

The patient is admitted in First Surgery Clinic of the Emergency Clinical County Hospital Târgu Mureş for malaise, abdominal pain, flatulence, weakness and fatigue. The physical exam noted a distended abdomen, tenderness and a hard, painful and poorly delimited tumor in the upper abdominal quadrant. Laboratory tests noted severe anemia (Hb: $6.75 \mathrm{~g} / \mathrm{dL}, \mathrm{Ht}: 22 \%$, serum Iron: $2.90 \mu \mathrm{mol} / \mathrm{dL})$ and hypoproteinemia (serum proteins: 5.70 $\mathrm{g} / \mathrm{dL}$ ). After a brief preoperative preparation, the patient underwent surgery under general anesthesia with endotracheal intubation.

Operative exploration revealed multiple tumors located in different organs: abscessed tumor with abdominal wall invasion on the right colon, tumor of transverse colon with transverse mesocolon invasion, tumor in the stomach with spleen invasion, sigmoid tumor and multiple peritoneal metastasis in the vicinity of sigmoid tumor. We performed a subtotal colectomy with ileo-rectal end-to-end anastomosis, a $2 / 3$ distal gastric resection with gastro-duodenal end-to-end anastomosis, splenectomy (Fig. 2, 3), and citoreductive pelvic peritonectomy for the peritoneal macrosopic metastasis. The surgical procedures were performed using electrothermal bipolar vessel sealing devices.

The postoperative course was uneventful. The patient was discharged in the $24^{\text {th }}$ postoperative day.

The histopathological exam noted massive infiltration of tumor cells in the colon and gastric wall with the presence of serous adenocarcinoma (Fig. 4) and immunohistochemical positivity to cytokeratin 7 (CK7) (Fig. 5) and negativity for cytokeratin 20 (CK20). Similar aspects 
were found in lymph nodes and peritoneal metastasis (Fig. 6, 7).

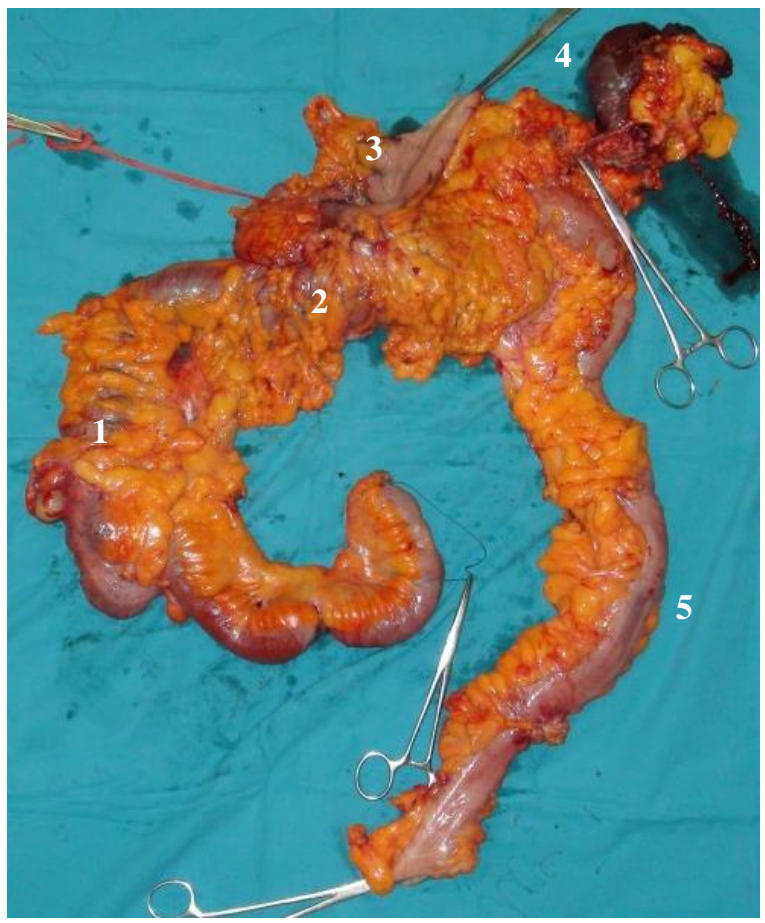

Fig. 2 Resected specimen (right colon (1), transverse colon (2), stomach (3), spleen (4), left colon (5)

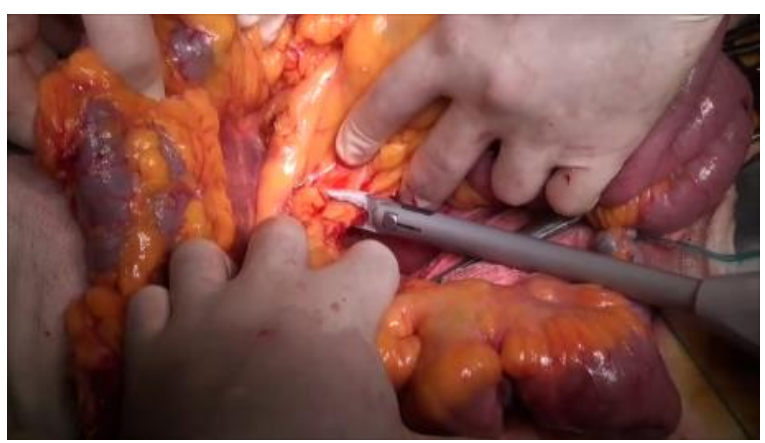

Fig. 3 Left colon resection with inferior mesenteric division using Ligasure ${ }^{\circledR}$ device

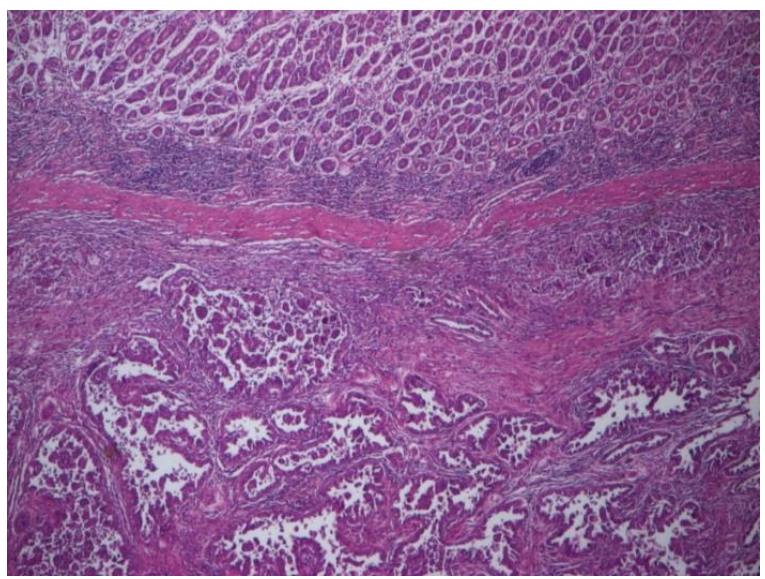

Fig. 4 Microscopic appearance of infiltrated gastric wall by atypical cells, glandular and papillary structures (hematoxilin eosin ob x 40)

\section{DISCUSSION}

Incidence of uterine papillary serous endometrial cancer is $5-10 \%$ from whole endometrial hystological types [7]. Colon metastasis of endometrial adenocarcinoma are very rare, and most commonly are the result of direct contact or peritoneal seeding than hematogenous or lymphatic dissemination. These are discovered usually in the form of serous membrane implants than of intraluminal tumors [8]. In our observation the tumor had a macroscopic appearance suggestive for a primary synchronous carcinoma of the colon (three different locations) with gastric invasion in the antrum and corpus. Only the colonoscopic biopsies suggested the relationship between serous endometrial adenocarcinoma and the present. Data from the literature regarding the spread of endometrial neoplasms suggests that secondary determinations to the colon are very rare, and often associated with endometriosis or peritoneal dissemination, consequence of an advanced endometrial cancer $[9,10]$. The present case shows no signs of endometriosis (clinically and histologically), and hystopathological protocols excluded a peritoneal carcinomatosis in the first presentation which shows once again its particularity.

Sohaib and Houghton, in a study conducted on a total of 86 patients with relapsed endometrial adenocarcinoma, revealed no cases of metastasis to the colon, the main target organs were the lymph nodes $(48 \%)$, vagina $(42 \%)$, peritoneum $(27 \%)$, lungs (27\%) and rectum (3\%) [1]. Yantiss and Clement shows 14 cases of malignant tumors in the colon with an endometrial starting point but with coexisting endometriosis [9].

Michael J. Anstadt and Shawn R. Lapetino proved that colorectal and endometrial adenocarcinoma have a number of morphological similarities, but the immunohistochemical profiles are different: the endometrial adenocarcinoma is CK7 positive, ER (estrogen receptor) positive, CK20 negative, and CDX-2 negative, 
whereas colorectal adeno-carcinoma is always positive for CDX-2, mostly CK20 positive and negative for CK7 and ER, these features being also confirmed in our observation. [8]

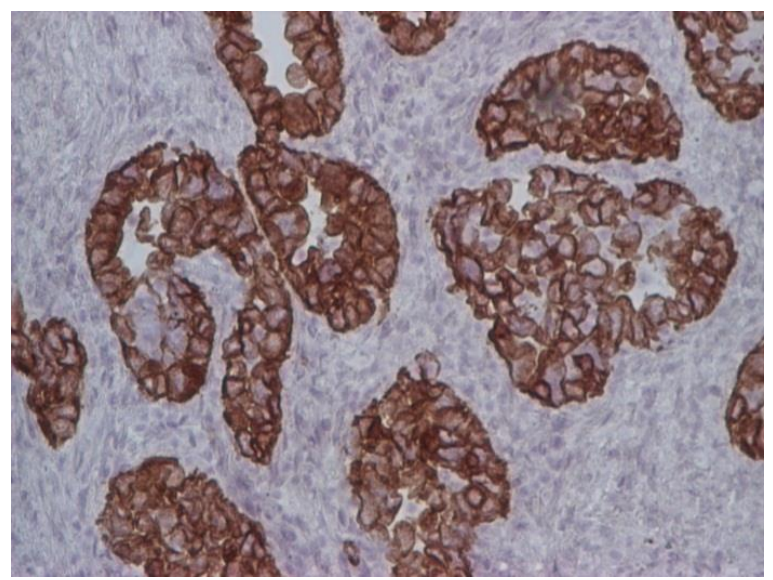

Fig. 5 Immunohistochemistry: tumoral cells positive to citokeratine 7 staining ( $\mathrm{ob} \times 200$ )

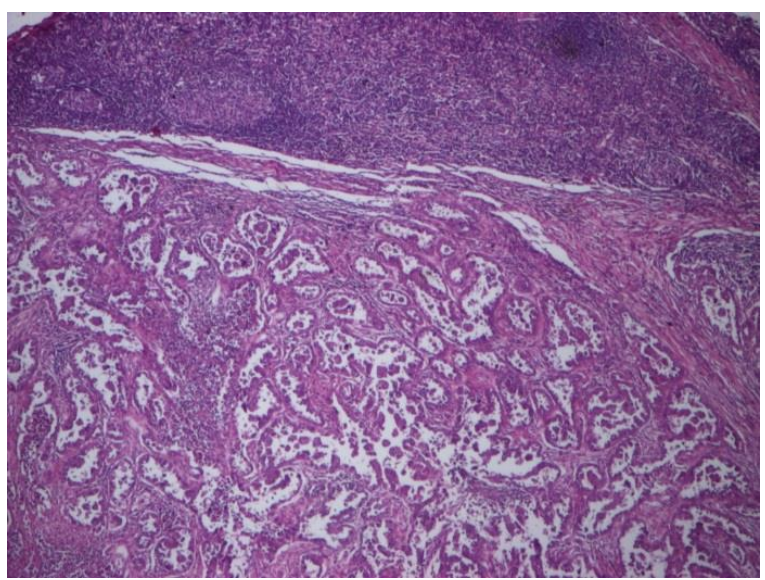

Fig. 6 Lymphonodes metastasis of serous adenocarcinoma (hematoxilin eosin ob x 40)

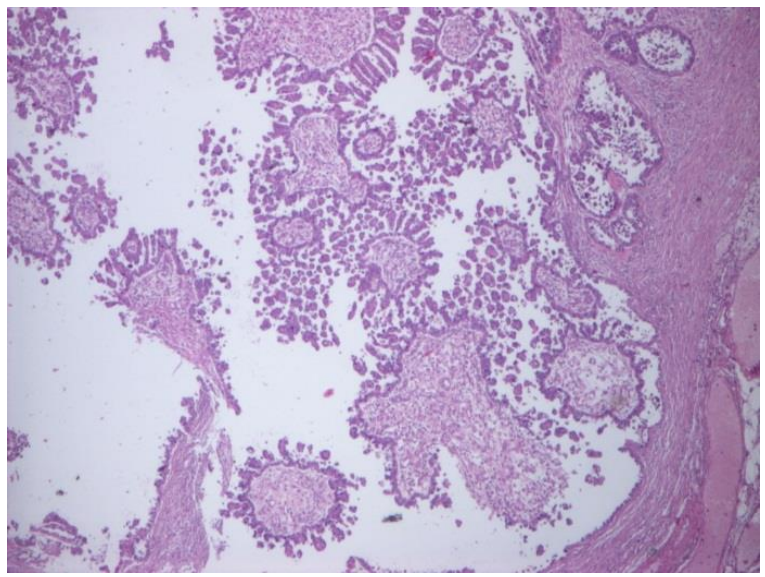

Fig. 7 Omental metastases: fatty-connective tissue infiltrated by papillary tumoral structures with connective-vascular axis adenocarcinoma (hematoxilin eosin ob x 40)
Recurrence of endometrial cancer involved in this case three colonic segments (ascendent, transverse and sigmoid), the distal part of the stomach and pelvic peritoneum. The surgical procedure was performed with electrothermal vessel sealing devices, wich reduced operation time, intraoperative bleeding and postoperative lymphoragia, and allow "en bloc" resection with minimal septicity.

\section{CONCLUSIONS}

The particularity of the presented case regards an infrequent progression of an endometrial adenocarcinoma correctly treated (oncologic resection followed by adjuvant therapy): synchronous gastric and multiple colic metastasis developed 4 years after the primary tumor diagnosis and treatment. The clinical appearance initially pleaded for primary synchronous cancers of gastrointestinal tract. Multivisceral resections were performed and the histopathologic exam confirmed the endometrial adenocarcinoma metastasis. The pathogenic mechanism of colic and gastric metastasis remains obscure in this case because the patient didn't have endometriosis or peritoneal carcinosis.

\section{CONFLICT OF INTEREST}

Authors have non conflict of interest to declare.

\section{REFERENCES}

1. Sohaib SA, Houghton SL, Meroni R, Rockall AG, Blake P, Reznek RH. Recurrent endometrial cancer: patterns of recurrent disease and assessment of prognosis. Clin Radiol. 2007; 62(1): 28-34.

2. Kirk GR, O'Rourke D, Ashe R, Clements WDB. Small bowel intussusception in metastatic endometrial carcinoma. The Ulster Medical Journal. 1999; 68(2): 110-113.

3. Thijs WJ, Karrenbeld A, van der Zouwen L, de Haan L. Metastatic endometrial cancer: a rare intestinal localization. Endoscopy. 2007; 39 (Suppl 1): E131.

4. Chen KT. Endometrioid adenocarcinoma arising from colonic endometriosis mimicking primary colonic carcinoma. Int J Gynecol Pathol. 2002; 21(3): 285-258. 
5. Oshiro H, Miyagi Y, Kawaguchi Y, et al. Endometrial adenocarcinoma without myometrial invasion metastasizing to the pancreas and masquerading as primary pancreatic neoplasm. Pathol Int. 2008; 58(7): 456-461.

6. Andrei S, Preda C, Andrei A, et al. Isolated splenic metastasis of endometrial adenocarcinoma - a case report. Chirurgia (Bucureşti) 2011; 106(6): 833-837.

7. Plataniotis G, Castiglione M, ESMO Guidelines Working Group. Endometrial cancer: ESMO Clinical Practice Guidelines for diagnosis, treatment and follow-up. Ann Oncol. 2010; 21 Suppl 5: v41-v45.
8. Anstadt MJ, Lapetino SR, Defnet A, Kapur U, Shoup M. Endometrial adenocarcinoma metastatic to the colon masquerading as a primary colon cancer. Journal of GHR. 2012 ; 21, 1(3): 40-43.

9. Yantiss RK, Clement PB, Young RH. Neoplastic and preneoplastic changes in gastrointestinal endometriosis: a study of 17 cases. Am J Surg Pathol. 2000; 24(4): 513524.

10. Hendrickson M, Ross J, Eifel PJ, Cox RS, Martinez A, Kempson R. Adenocarcinoma of the endometrium: analysis of 256 cases with carcinoma limited to the uterine corpus. Pathology review and analysis of prognostic variables. Gynecol Oncol. 1982; 13: 373-392. 
